\title{
Educación primaria: estrategias de intervención en contextos multiculturales
}

Concepción Hernández Noguera

Maestra de Educación Primaria en el CEIP "León Sola"

\section{Cuestiones previas}

Antes de centrarnos en estrategias concretas que podemos utilizar para facilitar el aprendizaje del alumnado bilingüe en contextos multiculturales, deberíamos de realizar una reflexión previa que nos ayudara a posicionarnos respecto a determinados conceptos claves en este campo.

\section{a) Educación multicultural-educación intercultural}

Una y otra educación tienen lugar en un contexto donde conviven grupos nacionales o étnicos diferenciados. Ambas toman en consideración el hecho de la diversidad, con el reconocimiento de los derechos de las minorías.

La educación multicultural respeta la diferencia, pero no incorpora los elementos enriquecedores de la diversidad al conjunto de los grupo sociales.

La educación intercultural supone un paso más, al rechazar el predominio de unas culturas sobre otras y defender que los distintos grupos que conviven en las actuales sociedades multiculturales pueden alcanzar una interdependencia enriquecedora basada en la valoración y el reconocimiento mutuo. 


\section{b) Asimilación-integración-separación-marginación}

Integración: Se produce integración cuando se conserva la identidad cultural y las costumbres y se buscan y valoran las relaciones positivas.

Asimilac:ón: Se produce asimilación cuando no se conserva la identidad cultural y las costumbres pero se buscan y valoran las relaciones positivas.

Separación: Se produce separación cuando se conserva la identidad cultural y las costumbres, pero se evitan las relaciones positivas.

Marginación: Se produce marginación cuando ni se conserva la identidad cultural y las costumbres ni se favorecen las relaciones positivas

Nuestra posición, como profesionales de la educación, respecto a estos conceptos previos debería de acercarse a la educación intercultural y la integración.

\section{c) Igualdad de oportunidades-situaciones iniciales de desigualdad} La igualdad de oportunidades es un principio que se propone luchar contra la desigualdad social ante la educación. Una puesta en práctica de este principio supone la posibilidad real de que todos puedan acceder al sistema escolar $y$, sobre todo, que todos tengan opción a obtener igualmente el éxito, de llegar al final, de triunfar. A partir de este presupuesto, se alega la necesidad de actuar en el entorno social, en la escuela, en la familia... para el logro de una auténtica igualdad, compensando las desigualdades iniciales, incidiendo en aspectos tales como la adquisición de competencias comunicativas, de aprendizajes instrumentales básicos, de desarrollo cognitivo, de habilidades sociales, etc.

Sin embargo, son los alumnos pertenecientes a minorías étnicas y culturales los que tienen tasas más altas de fracaso escolar; es decir, son los que están más representados en el absentismo, las repeticiones, el abandono temprano del sistema educativo, etc. ¿Por qué se produce esa separación tan pronunciada entre la declaración de principios sobre la igualdad de oportunidades y estos resultados escolares tan deficitarios?

\section{Análisis de la realidad educativa de Melilla}

Si queremos hacer una realidad el principio de igualdad de oportunidades, sin que éste entre en conflicto con las demandas de la educación intercultural y la integración, hemos de considerar las situaciones de partida desiguales que encontramos en nuestras escuelas. Estas son:

- La lengua oficial es el español. El aprendizaje de todas las áreas se basa en un adecuado manejo de esta lengua. Si su dominio es bajo (como es el caso del alumnado bilingüe desequilibrado), la situación inicial de desigualdad se hace manifiesta. ¿Cómo podemos conjugar la adquisición de las destrezas lingüísticas necesarias con el respeto a la lengua materna de nuestros alumnos y alumnas? 
- Los textos editoriales, los materiales curriculares del mercado están adaptados a una realidad social y cultural "estándar" y "occidental" como respuesta a las demandas del currículo oficial ¿Qué ocurre con la realidad concreta de nuestra ciudad diversa y "diferente"?

- Si a las diferencias lingüísticas y culturales se le suman situaciones socioeconómicas desfavorecidas, las dificultades para hacer del principio de igualdad de oportunidades una realidad se multiplican.

Estos tres aspectos derivan en las tres principales dificultades que encontramos en el proceso de enseñanza-aprendizaje de nuestro alumnado:

\section{A. Dificultades que proceden de las diferencias lingüísticas}

En el caso del alumnado cuya lengua materna es el tamazight, nos podemos encontrar con dos tipos:

- Alumnos bilingües, con un conocimiento equilibrado tanto del tamazight como del español. Las dificultades que presenten estos alumnos en su proceso de enseñanza-aprendizaje irán asociadas a otros aspectos ajenos a las competencias lingüísticas (capacidades, intereses, motivación, atención...)

- Alumnos bilingües desequilibrados o equilingüistas relativos, con un dominio mayor, generalmente, de la lengua materna.

Nos centraremos en este segundo grupo. Al reflexionar sobre cómo podemos aumentar sus competencias lingüisticas en español, no encontramos modelos que nos sirvan como referente.

- No podemos hablar de inmersión lingüística (caso de los inmigrantes), ya que en ese caso, la nueva lengua está presente en casi todos los ámbitos: en la calle, en el colegio (tanto por parte de los profesores como de los compañeros), en el barrio, en la tele... La lengua materna se reduce al entorno familiar. El alumnado, en este contexto, por lo general, adquiere rápidamente un dominio adecuado de la nueva lengua.

- No se trata de aprender una "lengua extranjera" (aunque algunos niños lleguen a la escuela sin ningún conocimiento del castellano] tal y como se concibe en el currículo escolar la enseñanza de esa segunda lengua.

- Los alumnos no viven en un entorno bilingüe absoluto: no existe el mismo peso de una lengua que de otra. El español se reduce al horario escolar ( 5 horas diarias a las que se restan los periodos vacacionales y los fines de semana) en el que conviven las dos lenguas (ya que, normalmente, en las conversaciones espontáneas la lengua utilizada es la materna). En el entorno familiar y social se utiliza mayoritariamente la lengua tamazight.

Teniendo como referente estos planteamientos, nuestra actuación docente debe centrarse en la siguiente premisa compaginar la enseñanza de la lengua oficial, el español, con el respeto a la lengua materna. Para ello, tendremos que:

- respetar las conversaciones espontáneas en la lengua materna, no debemos reprimirlas (especialmente, en los más pequeños], ya que podríamos crear conflictos de carácter diverso: afectivos, de autovaloración de la propia lengua y de la cultura... 
- partir del vocabulario en la lengua materna cuando no se conoce la palabra en español. Lo importante es el concepto, la dificultad mayor estriba en no conocer la palabra en ninguna de las dos lenguas. Para ello, el profesor (que normalmente no conoce el tamazight) puede apoyarse en la imagen. Es una fuente de motivación importante partir de la propia lengua, una llamada de atención, el alumno se siente valorado y tranquilo, especialmente, si el maestro se esfuerza en repetir esa palabra en la lengua del alumno.

- dar una gran importancia al lenguaje oral, sobre todo en los primeros años de escolaridad, potenciar el debate, el intercambio de opiniones, la escucha atenta,... empleando las técnicas y los recursos propios de este lenguaje.

- aconsejar a los padres que utilicen referentes lingüísticos distintos: el que posea un dominio adecuado del español, puede utilizar como medio de comunicación esta lengua (incluyendo la respuesta del niño), el que posea un mayor dominio del tamazight, utilizará esta otra. Ello permitirá que el niño desarrolle un uso correcto de ambas.

En otro apartado, nos centraremos en las técnicas y recursos concretos para aumentar las competencias lingüísticas.

\section{B. Dificultades que se derivan de las diferencias culturales}

Los niños de los distintos grupos culturales reciben un conocimiento en sus familias diferente del que encuentran en la escuela explicitado y adquirido a través de diferentes pautas de comportamiento, motivación y valores; actitudes y estrategias educativas, estilo de enseñanza, disciplina. La distancia entre la cultura de la familia/comunidad y la oficial de la escuela provoca conductas y actitudes en los alumnos calificadas de inadaptadas.

Cuando la distancia cultural con relación a la escuela es considerable, en el proceso de aprendizaje, puede aparecer una distancia cognitiva excesivamente elevada que reduzca las probabilidades de éxito en el alumno. Esta distancia cultural se traduce en tres planos: a] los contenidos, casi completamente ajenos a los conocimientos que posee el niño originariamente; $b$ ) los formatos, al utilizar modos casi exclusivamente verbales y abstractos, prescindiendo de las referencias de orden práctico o utilitario; c)

la relación educativa, en la medida en que el aprendizaje escolar tiene lugar en el marco de un sistema de comunicación pedagógica que presupone, por parte del alumno, el dominio (inexistente) de un tipo determinado de habilidades internacionales (por ej.. expresivas] y lingüísticas.

Pese a la declaración de intenciones, las propuestas y orientaciones educativas mediante las que se ha desarrollado la LOGSE, aún optando por medidas respetuosas de la diversidad, son especialmente ambiguas $y$, a veces, contradictorias cuando se refiere al tratamiento de las diferencias culturales. El Diseño Curricular Base por su generalidad y ámbito de aplicación no propone líneas de actuación específicas, pero el modelo de identificación cultural que subyace es restrictivo y restringido. Se deja en manos de las adaptaciones curriculares, orientadas hacia objetivos de aprendizaje y según modelos de déficit con planteamientos compensatorios, el tratamiento de la diversidad cultural. La Educación Multicultural aparece recogida como una dimensión de uno de los temas transversales en Primaria y Secundaria (la Educación para la Paz). Quedan ausentes cuestiones como la interacción cultural, la contextualización y relati- 
vismo cultural, la identificación social y asumir como objetivo educativo la construcción de significados culturales para todos desde planteamientos interculturales.

La realidad educativa que encontramos relativa a estos aspectos es la que sigue:

- Los textos editoriales y, en general, los materiales curriculares editados no se adaptan a la diversidad cultural y social de nuestros alumnos y alumnas.

- El análisis de las Unidades Didácticas (que habitualmente se desarrollan en uno $u$ otro ciclo) nos sugiere que los contenidos se trabajan desde una perspectivas monocultural evidente. De ello, encontramos múltiples ejemplos:

- La alimentación: caparecen alimentos o recetas de cocina típicos de otras culturas?

- La casa: edificio de pisos, casas con tejados o las diferentes y típicas viviendas de sitios lejanos. ¿Y las casas que aparecen en nuestra peculiar estructura urbanística?

- La familia: ¿Hay un único modelo de familia (padre, madre, hermanos/as, abuelo y/o abuela?

- Fiestas: ¿La Navidad, el Carnaval?

- Oficios: ¿Coinciden con los que encontramos en nuestro entorno próximo?

- Las estrategias de enseñanza aprendizaje deben fomentar un aprendizaje significativo y funcional, partiendo de la realidad, experiencias, vivencias, intereses de nuestros alumnos ¿Conocemos como docentes cuáles son estas realidades, experiencias, vivencias, intereses...? ¿Concuerdan con lo "ofertado" en el diseño de nuestro currículo? ¿Nos parecen suficientes y adecuados?

- La lengua que utilizamos como principal instrumento de comunicación constituye una dificultad, en algunos casos, más que una ayuda

- A todo esto, hay que añadir los llamados "dilemas culturales", las situaciones culturales conflictivas que encontramos en nuestras aulas. Ejemplos de estos dilemas nos encontramos a diario:

- En el caso de apostar por una educación en pro de la igualdad de la mujer, se podía acusar a la escuela de no respetar la cultura originaria, de imponer la cultura dominante; si por el contrario, se decide respetar tradiciones culturales, es posible también ser criticados por perpetuar en nuestro contexto la discriminación entre ambos sexos.

- Problemas semejantes pueden aparecer en áreas como conocimiento del medio al abordar tópicos como la educación corporal, los hábitos alimenticios... diferentes y algunas veces contrapuestos.

- Los propios planteamientos lingüísticos pueden ser otra fuente de conflicto: desde los que piensan que la lengua materna en estos niños obstaculiza el buen dominio de la lengua escolar hasta los que piensan que el currículo debería desarrollarse en lengua materna.

- Las celebraciones de algunas fiestas (tanto las propias de una cultura como de otra) así como otras (por ejemplo, el Carnaval) pueden levantar susceptibilidades y acarrear connotaciones negativas.

- El llevar el velo islámico (tema tan debatido actualmente) puede abrir una doble vía bajo el mismo principio de laicidad: la permisividad en nombre de la no-dis- 
criminación por razones religiosas y el conflicto ante la posibilidad de provocación proselitista.

Ante todos estos dilemas, lo primero que habría que subrayarse es la necesidad de que los profesores tuvieran una preparación mínima en aspectos como los siguientes:

a) conocimiento suficiente de los valores y comportamientos más relevantes de las culturas de sus alumnos. Esta precondición puede prevenir muchos conflictos interpersonales

b) formación de actitudes marcadamente abiertas que ayude a vivir los conflictos como algo no demasiado dramático en incluso enriquecedor, así como también de una orientación hacia las personas de los alumnos, más que hacia una tipificación de los mismos en los estereotipos adjudicados a su grupo de origen; esta última actitud supone, a su vez, una aceptación incondicional de un alumno determinado, un intento de solucionar los conflictos en tanto necesidades y problemáticas individuales.

En segundo lugar, en algunas ocasiones será inevitable el enfrentamiento entre ciertos valores tenidos como fundamentales por la cultura escolar y también como centrales por la cultura del alumno minoritario. El respeto no implica querer reconciliar lo irreconciliable. Cuando se dan actitudes discriminatorias en la educación familiar, la escuela, no puede. aunque sea veladamente, consentirlo, sea cual fuera la comunidad en que se produzcan. Una escuela que comulgue sinceramente con los valores del pluralismo auténtico tendría una medida común para juzgar tanto las desviaciones del grupo dominante (por ejemplo. actitudes de intolerancia, rechazo, xenofobia...) como la de otros colectivos minoritarios (por ejemplo, diferente dignidad de chicos y chicas fuera y dentro de la escuela). Hay una poderosa convicción de que existen unos valores universales a los que la naturaleza humana -y en su nombre cada cultura - tiende a dar asentimiento, directa o indirectamente. Teniendo en cuenta todo lo anterior, se comprende que la escuela ha de salir de una posición ambigua e insegura respecto a los múltiples dilemas. Se hace preciso, por un lado, una labor de reflexión por parte del profesorado sobre el papel que le corresponde como institución social y, por otro lado, acerca del ideario de la escuela en tanto portadora y transmisora de unos valores determinados

C. Dificultades derivadas de condiciones socioeconómicas deficitarias

La imbricación entre variables culturales y variables socioeconómicas es evidente y compleja. Frecuentemente, las dificultades para delimitar, diseñar y aplicar las propuestas educativas multiculturales se derivan de la íntima conexión marginalidad cultural y marginación social definitoria de la situación de muchos grupos culturales minoritarios que ocupan niveles sociales y económicos desfavorecidos en el entramado social. No es excepcional que las diferencias culturales enmascaren desigualdades derivadas de la situación social.

La pertenencia a grupos en situación de deprivación social y/o económica va a influir en muchos aspectos: valoración que se hace de la educación. grados de aspiración inferiores, tensiones percibidas por los niños en cuanto al diferente nivel que viven en la escuela y en su hogar (poco propicios para alcanzar altas cotas educativas), desiguales posibilidades económicas, distintos valores, intereses y vivencias... 
Desde nuestra planificación escolar, debemos tener en cuenta todos estos condicionantes. Los temas transversales pueden y deben jugar un papel importante en nuestro diseño curricular si queremos compensar algunos de los aspectos deficitarios y fomentar una verdadera educación en valores. Temas tales como la Salud, la Educación Medioambiental, la Educación para la Paz, la Coeducación... deberán introducirse, de manera continuada, en todas las unidades didácticas.

\section{Intervención educativa}

Situamos nuestra experiencia en el CEIP León Solá.

Nuestro centro está calificado como de difícil desempeño, presentando una serie de características que son las que dan sentido a nuestro trabajo.

\section{Análisis de la zona}

En relación al medio urbano en el que se enclava el centro, nos encontramos:

- Condiciones urbanísticas poco adecuadas: calles estrechas, sin asfaltar, sin aceras, con deficiente alumbrado y casi ninguna zona verde. El desorden urbanístico es patente ya que se observan casas edificadas arbitrariamente.

- Viviendas deficitarias en donde conviven un número elevado de personas.

- Edificios públicos, industriales y comerciales, escasos.

- Áreas culturales y recreativas casi nulas.

En relación al medio social, a grandes rasgos, detectamos:

- Una gran densidad de población.

- La organización social de la zona se caracteriza por pocas instituciones públicas y privadas, así como organizaciones administrativas y asociaciones culturales.

- Se trata de una zona de conflictividad social donde conviven familias con escaso poder adquisitivo, alta tasa de desempleo y un número elevado de miembros dentro de cada unidad familiar.

En relación al medio familiar:

- Bajo nivel socio-económico traducido en carencias físicas y condiciones precarias con influencias negativas en el desarrollo escolar, ya que la salud del niño/a, el origen y proceso de la fatiga, física e intelectual, la higiene, etc, son factores de tipo fisiológico que en la dinámica escolar han de tenerse en cuenta.

- Vivencias muy reducidas: el alumnado se mueve, casi exclusivamente, en el entorno familiar, en el barrio. Sus experiencias vitales son escasas ya que el ambiente es pobre en estímulos.

- Poca valoración e interés, por parte de las familias, hacia lo escolar.

- La propia estructura familiar, con un número elevado de hijos e hijas, puede repercutir en la atención que se presta a cada uno de ellos. De igual manera influyen los distintos roles adoptados por la madre y el padre, así como el tiempo en que cada uno de ellos permanece en casa. 
- Modelos familiares inestables, a veces como consecuencia de la diferencia de roles, en los que se mezcla un excesivo autoritarismo con una flexibilidad extrema. La mezcla de condescendencia y restricción provoca conductas agresivas en el niño/a ya que éste se ve persistentemente sometido a condiciones que fomentan sentimientos de hostilidad y al mismo tiempo no le imponen límites a su comportamiento decidido cuando expresa esta hostilidad.

- Falta de actuaciones coordinadas entre las familias y la escuela ante problemas de disciplina y problemas académicos, lo que puede acarrear en el alumno tensiones internas por no saber cómo actuar, fomentándose la creación de actitudes negativas.

- Analfabetismo funcional en un número elevado de padres y/o madres. Ello se traduce en una escasa participación de las familias en la dinámica escolar.

\section{Orientaciones para la planificación didáctica}

La intervención didáctica debe adaptarse a las circunstancias especiales de cada grupo de alumnos, ya que la diversidad ha de ser afrontada de manera diferente según el entorno en el que se muevan.

El objetivo final de la Educación Intercultural es promover un encuentro entre diferentes colectivos, grupos étnicos, etc., donde se produzca un intercambio en pie de igualdad, conservando la especificidad de cada uno, al tiempo que buscando el enriquecimiento mutuo.

La educación intercultural pretende que la sociedad autóctona ["mayoría"):

- Conozca y modifique los estereotipos y los prejuicios que tiene de los diferentes grupos de la minoría.

- Favorezca el conocimiento y la valoración positiva y crítica de las culturas minoritarias.

- Propicie una toma de conciencia sobre la necesidad de un mundo más justo.

- Promueva actitudes, conductas y cambios sociales positivos que eviten la discriminación y favorezcan las relaciones positivas, posibilitando el desarrollo especifico de las culturas minoritarias.

Así mismo, promueve el que las minorías étnicas:

- Conozcan y modifiquen los estereotipos y los prejuicios que tienen de la mayoría.

- Favorezcan el conocimiento y la valoración positiva y crítica de las culturas mayoritarias.

- Den a conocer la propia cultura.

- Promueva actitudes, conductas y cambios sociales positivos que eviten la discriminación y mejoren sus condiciones de vida.

Existen objetivos comunes para las mayorías y las minorías pero se presentan de forma separada para dejar claro que la Educación Intercultural implica a todas las partes. Para alcanzar estos objetivos, el equipo educativo debe reflexionar, discutir y consensuar las estrategias didácticas concretas que se van a adoptar en la práctica cotidiana. 
He aquí algunas de las orientaciones que pueden ser utilizadas como referentes en la planificación didáctica.

- Diseñar actividades motivadoras y gratificantes.

- Partir de las experiencias y conocimientos previos del alumnado.

- Utilizar gran variedad de métodos didácticos, porque parece demostrado que los alumnos con desventajas rinden más cuando se les da una enseñanza diversa y novedosa.

- Reconocer activa y explícitamente los valores culturales de los diferentes grupos.

- Introducir en el currículo escolar contenidos de las diferentes culturas

- Conocer lo más posible a los alumnos, tanto en intereses, forma de vida, vivencias; saber "diagnosticar" sus necesidades para poder partir de sus propias experiencias en la intervención educativa.

- Analizar "materiales" adecuados para una educación intercultural, así como elaborarlos y/o utilizarlos.

- Buscar el equilibrio entre el derecho a la diferencia y la igualdad de oportunidades.

- Transformar cualitativamente el currículo acercando sus contenidos y actividades a la cultura familiar, eliminando conflictos pero sin que ello suponga reducir los objetivos educativos.

\section{Estrategias de intervención}

A la hora de establecer unas estrategias concretas de intervención, es fundamental partir del contexto educativo concreto en el que nos hayamos inmersos. Teniendo a éste como referente, nos centraremos en cuatro propuestas para trabajar en el aula:

- El cuento

- El juego

- La introducción de contenidos culturales en las diferentes unidades didácticas

- Criterios de globalización e interdisciplinariedad como respuesta metodológica.

\subsection{El cuento}

Muchos de nuestros alumnos presentan unas caracteristicas lingüisticas muy peculiares: escaso dominio del vocabulario básico, construcciones sintácticas y semánticas deficitarias, regularización errónea de las formas verbales, sustitución de verbos ("hacer" por "poner", "hacer" por "dar"...), empleo incorrecto de nexos lingüisticos, etc. A esto hay que añadir que en su lengua materna aparecen vocablos únicos para definir algunos conceptos que en castellano aparecen claramente diferenciados.

Como consecuencia, el desarrollo funcional del lenguaje (que influye de modo determinante en la estructuración de su pensamiento) es lento y desajustado al currículo escolar. La lengua ha de estar presente en la escuela como instrumento para representar y comunicar, para obtener y ofrecer información. Es un instrumento fundamental para cualquier experiencia de aprendizaje y conocimiento de la realidad. 
Es función de la educación primaria el incremento y diversificación de las destrezas comunicativas del alumnado, de modo que el uso oral y escrito se enriquezca por el estímulo realizado desde el ámbito escolar.

En este sentido, el cuento, por sus propias caracteristicas (motivante, capaz de centrar la atención, lúdico, ...) es un buen recurso en estas edades para trabajar en una doble vía:

- los dos procesos básicos de la comunicación: el de expresión y el de comprensión

- actitudes y valores

\section{Cómo trabajar el cuento}

Son muchos los modos de trabajar el lenguaje a través del cuento. Es importante que las estrategias sean variadas (para que el cuento no pierda el carácter lúdico) y participativas (que permitan la actuación del alumnado, de manera que les ayude a centrar la atención). He aquí algunas de ellas.

a) Contar un cuento. El objetivo es que los alumnos disfruten de la lectura (es recomendable que la lectura la haga el maestro/a ya que en la lectura colectiva se está más pendiente de "seguir la línea" que del contenido en sí de lo leido). Aquí, es muy importante la elección del cuento, debe ser apropiado a la edad e intereses de los alumnos.

b) Contar el cuento abriéndolo a la participación del que escucha: dejar frases "evidentes" sin terminar (érase una...), ponerle nombre a los personajes (una niña que se llamaba...), introducir alguna "mentira" que han de descubrir (el lobo era bondadoso). El objetivo es centrar la atención, "enganchar" al alumno en la lectura del cuento.

c) Trabajar el vocabulario a través del cuento. No se trata de simplificar excesivamente el cuento para que lo entiendan sino de enriquecer y mejorar las destrezas comunicativas del alumnado a través de él. La clave estaría en aprender palabras nuevas y hacer que los alumnos las utilicen para así incorporarlas a su bagaje. Para ello, es adecuado utilizar un cuento que continúa o, incluso, volver a contar uno ya contado (permite recordar las palabras aprendidas). Una posible actividad para utilizar el nuevo vocabulario sería: escribir una parte del cuento, completando frases con "datos" del cuento y con las palabras aprendidas (se puede utilizar algún tipo de ayuda, dependiendo de la edad del alumnado y de la complejidad de las nuevas palabras, como, por ejemplo. hacer un listado con todas las posibles).

d) Lectura individual de un cuento. Después de ella, se pueden realizar múltiples actividades:

- Verbalización posterior, libre o dirigida por el maestro/a.

- Si el cuento es el mismo para todo, escenificar parte del cuento.

- Descripción de personajes

- Buscar títulos

- Cambiar el final

- Poner condicionantes al cuento (¿qué pasaría si...?) que normalmente se utilizan en la formación de actitudes o búsqueda de valores

- Preguntas sobre los sucesos del cuento. 
e) Reescribir un cuento. Para ello:

- Verbalizar, individualmente o en equipo, las secuencias temporales

- Escribir frases para cada una de esas secuencias.

- Ponerle título

- llustrarlo

f) Escribir un cuento. En las primeras fases, es necesario, hacerlo de manera colectiva en el grupo-clase organizando previamente lo que se va a desarrollar. Ello supone:

- Buscar personajes principales, describirlos y caracterizarlos

- Buscar un escenario donde ubicar la acción y los personajes

- Buscar un hilo conductor, pensar una secuencia sencilla de acciones.

- Buscar posibles desenlaces.

- Elegir un título.

\subsection{El juego}

Otro recurso importante para trabajar los proceso de comunicación y fomentar actitudes positivas es el juego, especialmente en el primer ciclo de Educación Primaria.

En contextos multiculturales y bilingües es importante que el juego reúna, además de las características que le son propias, las siguientes:

- que conlleve la utilización del lenguaje como elemento clave (a través de la ampliación de vocabulario, siguiendo instrucciones determinadas, planteando cuestiones relativas a la unidad que se esté desarrollando...]

- que sea cooperativo, potenciando el trabajo en equipo, la interdependencia, y haciendo un análisis posterior que permita valorar la actuación de cada equipo o de sus miembros

- que potencie actitudes coeducativas, de colaboración y de respecto hacia los demás.

\section{Algunos tipos de juegos}

Por un lado, podemos recurrir a los típicos del lenguaje: palabras encadenadas, sopas de letras, adivinanzas, trabalenguas...

Por otro, a un amplio abanico que va desde aquellos relacionados con la unidad didáctica en curso (juegos más relacionados con el área de conocimiento del medio) hasta otros de carácter más general: juegos de mímica, de interpretación, de habilidad, de memoria, de imaginación....

Para concretar este apartado, nos centraremos en los segundos (ya que los primeros son suficientemente conocidos], poniendo dos ejemplos concretos.

\section{El juego de los sentidos (UD El Cuerpo)}

Se trata de un juego de preguntas sobre los cinco sentidos.

El material utilizado comprende: un fichero con tarjetas (en cada tarjeta aparecerán cinco preguntas, cada una de ellas relativa a un sentido y diferenciadas por un color), un dado gigante (de los utilizados en psicomotricidad), en cinco de sus caras hay una 
cartulina pegada de cada uno de los colores representados en las tarjetas (cada color se corresponde con un sentido).

Antes de comenzar el juego, se divide la clase en grupos de cuatro alumnos (procurando que haya niños y niñas). Cada grupo dispone de un tiempo para buscar el nombre del equipo y elegir una capitán, que será el encargado de leer la pregunta que nos indique el dado y ser el portavoz del grupo. En la pizarra, se hace un cuadrante con el nombre de los distintos equipos. Previo al comienzo del juego, se explica a la clase que si algún grupo y/o miembro no respeta las normas del juego (responder cuando no le corresponde, hablar cuando lo hace otro compañero...J, se verá sancionado con un punto negativo.

Para comenzar, se reparte una tarjeta a cada equipo. Se tira el dado que nos indica el sentido al que hace referencia la pregunta. Cada equipo busca el color correspondiente y durante un minuto lee e intenta responder a la pregunta. Se comienza una ronda de respuestas. Si el equipo responde correctamente, se le anotan dos puntos. Si el equipo falla su pregunta, no se le anota ningún punto y el siguiente equipo puede contestarla en su lugar, anotándose en este caso un punto. Para la segunda ronda de preguntas, se vuelve a repartir una nueva tarjeta entre cada equipo y se vuelve a tirar el dado y así sucesivamente.

Al final del juego, se suman y se restan los puntos de cada equipo para saber quién es el ganador.

Por último (es muy importante), se analizan las distintas situaciones del juego y se plantean preguntas tales como: el equipo que ha ganado cha sido el que más preguntas ha acertado o el que no ha tenido ningún punto negativo (es decir, el que ha respetado las normas del juego)? ¿quién ha quedado segundo? ¿ha tenido algún punto negativo? Si no los hubiera tenido chubiera ganado?... Es importante incidir en dos hechos: por un lado. no saber una pregunta no es lo peor (ya que la puntuación es 0), lo peor es no respetar a los demás compañeros o las normas; por otro, es el propio grupo el que tiene que equilibrar la conducta de sus miembros si no quieren ser sancionados.

\section{Juego de habilidades múltiples}

El material utilizado es 5 juegos de cartas (de 25 cartas cada uno), correspondientes a: mímica, interpretación, memoria, habilidad, imaginación, y un dado.

En la primera ronda, cada jugador tira el dado y coge una carta del montón correspondiente al número que le ha salido. A continuación hace lo que indica la carta o contesta a la pregunta.

En una segunda ronda, se hacen dos grupos con los jugadores que han realizado la prueba correctamente. Esta vez, se juega con un tablero dibujado en la pizarra. Si el grupo supera la prueba, avanza las casillas que corresponden. Puede caer en una casilla que le obligue a retroceder o estar un turno $\sin$ tirar. Gana el equipo que llega antes al final del tablero.

Si la motivación no ha decaído, se pueden volver a hacer otros dos grupos con los alumnos que no realizaron, inicialmente, la prueba de forma correcta, dándoles una segunda oportunidad. 
Es muy importante, igual que en el juego anterior, hacer una valoración al final del juego, incidiendo en el hecho de que es mucho más fácil realizar una prueba de forma cooperativa.

\subsection{Introducción de contenidos culturales en las Unidades Didácticas}

Todos los alumnos tienen derecho a que el sistema educativo les ayude a potenciar conocimientos y habilidades que respondan a las necesidades derivadas de la diversidad cultural.

Los objetivos seleccionados y priorizados deberían abarcar todas las dimensiones de la educación multicultural y referirse tanto al conocimiento y comprensión de nuevos contenidos, como a la adquisición y cambio de actitudes, entrenamiento en habilidades técnicas y aplicación de nuevas conductas personales y sociales.

Por otro lado, si queremos que el aprendizaje de los alumnos sea significativo, debemos partir de las experiencias y conocimientos previos del alumno.

Todo ello nos conduce a la necesidad de introducir en nuestras unidades didácticas contenidos que atiendan a la diversidad cultural del aula en un doble sentido:

- Desde el punto de vista cognitivo: las costumbres, valores y creencias de las principales culturas representadas en el aula (conceptos] y la evaluación objetiva de la propia cultura (procedimientos)

- Desde el punto de vista afectivo: el sustrato humano que todos compartimos, los principios de igualdad de derechos y justicia social (actitudes, valores y sentimientos).

\section{La unidades didácticas y sus contenidos culturales y sociales}

Al hablar de introducir contenidos sociales y culturales en las distintas unidades didácticas, no debemos limitarnos a la celebración de las fiestas ni, en el caso extremo, intentar introducirlos en todas las unidades. No significa que todos los aspectos curriculares hayan de ser completamente congruentes con las prácticas culturales originarias de los alumnos; se trata de utilizar el conocimiento de cada cultura (y de los modos diferenciados de socialización que provocan) como una guia útil para seleccionar los programas educativos oportunos con objeto de conseguir los objetivos académicos queridos y evitar los no deseados. No se trata, pues, de una enseñanza pasiva y románticamente "adaptada" a todos y cada uno de los rasgos culturales propios de los alumnos, sino de una educación ajustada a tales diferencias, que potencia las compatibilidades y evita las incompatibilidades que puedan encontrarse entre los distintos polos de referencia.

Para facilitar el proceso de aprendizaje de los alumnos hay que partir de lo que el niño ya conoce, enriqueciendo sus conocimientos, aumentándolos y proporcionándoles las destrezas necesarias para que él mismo sea capaz de comprender la diversidad que no está a su alcance y que, desde luego, no podemos emplear como punto de partida en este proceso.

Por tanto, al tratar la familia, tendremos en cuenta la estructura familiar peculiar de cada alumno o de cada cultura o situación social, la convivencia en la casa, el número de elementos..., hasta llegar a otras estructuras familiares "diferentes". 
Al tratar del cuerpo, podemos encontrarnos con un conocimiento adecuado de las distintas partes del mismo en la lengua materna, no así en castellano. Eso no significa que el alumno no tenga adquirido el esquema corporal sino que debe aprender un vocabulario específico. Podemos encontrarnos, también, con connotaciones extrañas asociadas a la lateralidad que habrá que conocer y en lo posible, superar.

Al tratar la casa, debemos partir de "su casa", "su barrio", de la infraestructura urbanística en la que están inmersos y de la propia estructura familiar, para ampliarles, en un segundo término, estas perspectivas.

Al tratar la alimentación, introduciremos alimentos propios de las distintas culturas, recetas de cocina,... y valoraremos objetivamente los distintos alimentos como beneficiosos o perjudiciales aunque sean propios de una u otra cultura (por ejemplo, el café y el té, como pertenecientes a la misma categoría).

Al introducir un tema transversal como el medio ambiente, tendremos en cuenta que, a veces, hay que luchar contra situaciones sociales deprivadas que constituyen un verdadero handicap (en algunos barrios, por ejemplo, no hay papeleras que permitan cuidar nuestro entorno).

Al trabajar otro tema transversal, como la coeducación, nos podemos encontrar con actitudes cerradas y estereotipos que será necesario cambiar.

Al celebrar "nuestras" fiestas, "sus" fiestas, debemos hacerlo desde actitudes de respeto y de enriquecimiento mutuo: el conocimiento de las distintas culturas, costumbres y valores es importante, no debemos centrarnos en una sola y mucho menos en nuestra Comunidad de por sí diversa.

\subsection{Perspectiva globalizadora e interdisciplinar}

Introducir una perspectiva globalizadora e interdisciplinar en la enseñanza no es tanto una cuestión de técnica educativa como de la actitud con que enfrentamos el hecho educativo. Actitud que comporta entre otras cosas, introducir contenidos únicamente en el contexto experiencial del alumno y ser rigurosos en la aplicación de los distintos instrumentos de conocimiento, independientemente de la disciplina que los soporta.

La globalización se basa en un criterio psicológico: el niño percibe y conoce a través de captaciones de todos no diferenciados. Los contenidos se agrupan en torno a núcleos o centros de interés para el alumno, facilitando el aprendizaje significativo.

La perspectiva globalizadora no prescribe métodos sino que da pautas para organizar y articular los conocimientos en secuencias de aprendizaje orientadas a un fin concreto con sentido e intencionalidad y situadas en contextos que permitan su significatividad y funcionalidad.

La Interdisciplinariedad organiza los contenidos buscando elementos comunes a las distintas disciplinas: lenguaje común, conceptos, métodos e instrumentos, siempre que la naturaleza de los contenidos tratados lo recomiende. Temas como el clima, la salud, la educación vial, etc, no pueden ser comprendidos en profundidad a partir del análisis de una única disciplina, sino que requieren las aportaciones de distintas disciplinas, realizadas desde sus marcos teóricos y procedimientos propios. 
Globalización e interdisciplinariead son dos términos entendidos a veces como sinónimos y en ocasiones, paradójicamente, como antónimos. Estos términos no son comparables, ya que pertenecen a órdenes distintos. Globalización se refiere a cómo nos acercamos al conocimiento de la realidad y a cómo ésta es percibida y comporta una intencionalidad totalizadora en cuanto a los elementos que la componen. En cambio, las disciplinas y sus distintas formas de relacionarse no se refieren a cómo es la realidad, sino que su función es la de ofrecernos los medios e instrumentos para llegar a su conocimiento.

Adoptar esta perspectiva, supone cambios de diverso tipo:

- En relación a las programaciones, revisarlas y realizar los ajustes necesarios para adaptarlas, lo máximo posible, a la realidad escolar.

- En relación al alumnado, proporcionarles un aprendizaje experiencial, vivencial, vital, capaz de suplir las carencias del medio en el que se desenvuelven pero dotado de significatividad, intentando incidir en aspectos tales como motivación, interés, grado de participación....

- En relación a los métodos, enfrentar la tarea de presentar los objetivos y contenidos que deben alcanzar nuestros alumnos/as para optimizar su proceso de aprendizaje dentro de esta nueva perspectiva experiencial. Se trata de "repetir"conceptos sin aburrir; de incidir sobre todo en contenidos actitudinales sin olvidar o menoscabar conceptos y procedimientos; de elevar el protagonismo de los alumnos y la participación en su propio aprendizaje.

- En relación a los recursos, confeccionar materiales didácticos alternativos a los existentes y adaptados a las necesidades.

\section{Aportaciones de las distintas áreas}

La finalidad es conseguir los mismos objetivos pero trabajados desde diferentes perspectivas curriculares, aprovechando los recursos que ofrece cada disciplina para hacer el aprendizaje más significativo.

En el área de Educación Física, se aprovecha el carácter recreativo y motivador que, ya de por sí, caracteriza a éste área, tanto a través de juegos motrices como de otro tipo de actividades lúdico-educativas.

En el Área de Educación Artística, se trata de reforzar y complementar los contenidos del tema realizando actividades musicales y plásticas, tomando como punto de referencia esos mismos contenidos. Estos aparecen en canciones, dramatizaciones, etc, que se complementan, en algunos casos, con actividades rítmicas realizadas igualmente por el especialista de Educación Física.

En las Áreas de Lengua, Matemáticas y Conocimiento del Medio se trata de aprovechar los recursos propios del aula y el hecho de ser una sola persona la que las imparte. Ello permite incidir en diversos aspectos:

- Flexibilidad en la organización del tiempo

- Realización de distintos tipos de actividades: juegos de carácter cognitivo, concursos, simulaciones, análisis de situaciones... 
- Elaboración de fichas individuales adaptadas a los contenidos de la Unidad y de nuestra propia realidad.

- Confección, de forma cooperativa, de murales, dentro y fuera del aula.

- Plasmación escrita y gráfica de los contenidos tratados.

- Aprovechamiento de las actividades extraescolares como recurso didáctico.

\section{Medidas adoptadas}

Este planteamiento nos lleva, a grandes rasgos, a:

- Incrementar la coordinación entre todo el profesorado. Es necesario establecer pautas comunes de actuación, proceder a la nueva programación de actividades, distribuir tareas, aprovechar las aportaciones individuales y encauzarlas en un proyecto común.

- Realizar modificaciones puntuales en el horario.

- Organizar actividades que requieren la colaboración de otros profesionales, implicando no sólo al alumnado sino también a las familias.

- Elaborar y buscar nuevos recursos didácticos

- Ambiental y de referencia al tema a tratar, involucrando, en la medida de lo posible, al resto del centro y utilizando tanto los espacios comunes como los del aula (confección de franelogramas y mascotas).

- Recursos lúdicos, tales como puzzles, guiñol, teatro, cuentos, composiciones musicales, juegos motrices...

- Búsqueda de bibliografía y elaboración de fichas adaptadas a cada unidad y a cada nivel.

\section{Actividades tipo}

De todas las unidades didácticas recogidas en el Proyecto Curricular, hemos seleccionado tres que consideramos que son las más interesantes dadas las características de nuestro centro, nuestros alumnos y alumnas y su entorno.

- El Cuerpo y la Salud

- La alimentación

- Plantas, animales y Medio Ambiente.

A continuación exponemos un listado de actividades-tipo que desarrollamos en cada unidad. Queremos, previamente, hacer hincapié en dos aspectos:

- La mayoría de las actividades son diseñadas para uno u otro nivel, algunas son comunes para los dos (por ej., teatro) y otras aún siendo comunes varían en cuanto al grado de complejidad (poesías, cuentos...).

- Algunas actividades se realizan separadamente tutor-especialista y otras de forma conjunta. 
Unidad Didáctica:

El cuerpo y la salud

- Confección de mascotas: Don Limpio (un contenedor de basuras), El Cepillo Pillo (un cepillo de dientes) y las pompitas de jabón Plaf, Plef, Plif, Plof, Pluf.

- Franelogramas: Posturas correctas e incorrectas, acciones perjudiciales y beneficiosas para el cuerpo, accesorios para la higiene corporal y dental, un cuarto de baño... - Juegos motores y actividades lúdico-educativas en el Área de Educación Física: Juegos de corro (¿Cómo planta usted las flores?); juego del olfato; familias de elementos de higiene personal; el baile de las posturas correctas e incorrectas; caries, microbios y piojos; trabajos de respiración (pompas de jabón)...

- Trabajos grupales con paneles del cuerpo humano [partes del cuerpo, órganos internos]

- Juegos de los sentidos realizados por los tutores/as.

- Juego cooperativo sobre los sentidos ("el dado de los sentidos").

- Canciones: El hoky Poky; Veo con mis ojos, Mi cara; Moviendo el cuerpo, Dibujar mi cuerpo, Los dedos de mi mano; El baile de las articulaciones; Los sentidos; Un, dos tres; -El Cepillo Pillo: Plaf, Plef, Plif, Plof, Pluf, las pompitas de jabón; Levántate ya; Después de comer; Pim-Pom.

- Poesías: Lo que dice el espejo, la cara de Doña Sara; La Semana Limpia...

- Cuentos: Vicente el diente, Don Limpio, Las Cinco Pruebas...

- Guiñol sobre higiene dental: El Cepillo Pillo y la señora Doña Pasta

- Dramatización de situaciones sobre hábitos higiénicos (duchas, lavado de manos y dientes)

- Actividades de plástica: mariquitinas, completar dibujos de figuras humanas, porta-cepillos, móvil de pompas de jabón...

- Concursos: plastilina, dibujo, poesía, adivinanzas y los sentidos.

- Resolución de problemas reales o introducción de conceptos matemáticos aprovechando los recursos que nos brinda la Unidad (peso, medidas de altura...).

- Charlas de profesionales del Centro de Salud de Cabrerizas:

- Enfermeras, dirigida al alumnado

- Pediatra, dirigida a padres y madres

- Actividades sobre hábitos de higiene: Duchas en el gimnasio (tutor/a-especialista de Educación Física), lavado de manos antes de acudir al Comedor Escolar, lavado de dientes después del recreo.

- Visita al Centro de Salud de Cabrerizas. 
Unidad Didáctica:

La alimentación

- Confección de mascotas: Blanqui (una botella de leche)

- Franelogramas y decoración de pasillos: tiendas (frutería, pescadería, carnicería, panadería, chucherías], simulación de un supermercado, alimentos aconsejables y no aconsejables u ocasionales, muestras reales de distintos tipos de desayunos y de menús equilibrados (desayuno, almuerzo, merienda y cena).

- Juegos motores y actividades lúdico-educativas: Dramatizaciones sobre hábitos alimenticios y sus consecuencias; juego de rol (persecuciones) sobre alimentos sanos y las chucherías...

- Canciones: En el Mercado, Las frutas, La naranja se pasea (con coreografía), Si nos mandan a la tienda...

- Poesías: He perdido el apetito, Las frutas; Boda de Doña Sandía y Don Melón

- Cuentos: El desfile de los alimentos, La historia de Blanqui, La cadena de los alimentos...

- Trabalenguas sobre alimentos

- Recetas de cocina.

- Adivinanzas sobre los alimentos

- Desfile de los alimentos (disfraces)

- Actividades de plástica: Señalador de libros, cuaderno de recetas de cocina, confección de los disfraces para el desfile,...

- Concursos: Plastilina, dibujo, poesía, recetas de cocina, adivinanzas.

- Resolución de problemas reales o introducción de conceptos matemáticos aprovechando los recursos que nos brinda la unidad.

- Visita al Mercado de Cabrerizas

- Visita al Mercado Central

- Preparación colectiva de batido de frutas

- Preparación colectiva de macedonia de frutas

- Visita a la Panadería de Intendencia

- Elaboración de pan en el horno tradicional de adobe y desayuno en el aula

- Comida en el Comedor del Centro con la presencia de tutores (incidencia en el menú y en el uso correcto de los cubiertos), lavado de dientes posterior.

- Charlas sobre alimentación impartidas por:

- Enfermeras, dirigida al alumnado

- Pediatra, dirigida a las familias 
Unidad Didáctica:

Plantas, animales, medio ambiente

- Confección de mascotas: Don Limpio (un contenedor de basuras). El Árbol Sabio (un árbol que enseña comportamientos ecológicos]

- Franelogramas y decoración de pasillos: plantas; animales; "Nuestro Huerto", con distintos tipos de planta y su siembra; panel con fotos de algunas de las plantas de los Pinares de Rostrogordo; panel con fotos de algunas de las plantas del Parque Hernández;carteles medioambientales con pie de foto confeccionado por los alumnos/as; contenedores para la recogida selectiva de basuras, ilustraciones en papel continuo de nuestro cuento ecológico "El elixir mágico"...

- Juegos motores y actividades lúdico-educativas: Tragabolas (cajas de cartón decoradas y pelotitas de plástico o de papel aluminio). Esquí acuático (desplazamiento sobre cartones). Apagar el fuego (con botellas de plástico y envases de yogur), "los barrenderos"...

- Canciones: Cómo nace una planta

- Audición y expresión corporal de "Campanas del Monasterio" de Ketelbey.

- Cuentos: Cuentos sobre animales (El caracol feliz, El largo cuello de la jirafa, La araña y el ciempiés, La liebre y la tortuga....). Cuentos sobre el Árbol Sabio (colección de cuentos sobre comportamientos ecológicos). Una clase al aire libre, Cuatro estaciones hay en el año, Cuento ecológico "El elixir mágico".

- Cuento auditivo (montaje con soporte cassette): "El Árbol sin hojas"

- Obra de teatro: "El Árbol Sabio"

- Dramatización de situaciones: Cómo nace una planta.

- Actividades de plástica: Cuaderno de siembra (Nuestro Huerto), cuadernos de cartón con los itinerarios de las visitas y fichas a realizar durante las mismas, móviles de flores, construcción de una granja, trabajos de papiroflexia con animales...

- Concurso sobre construcción individual de juegos o trabajos con material de desecho.

- Premio al comportamiento más ecológico.

- Resolución de problemas reales e introducción de conceptos matemáticos aprovechando los recursos que brinda la Unidad.

- Reciclado de papel

- Elaboración casera de jabón

- Semilleros

- El Huerto

- Recogida colectiva de basuras del patio de recreos

- Elaboración de juegos colectivos con material de desecho.

- Visitas a los Parques Hernández y Lobera

- Excursión a los Pinares de Rostrogordo

- Acampada en el Centro 
Cada una de las unidades descritas anteriormente se llevan a cabo en cada uno de los periodos trimestrales (a lo largo del curso, se realizan actividades de recuerdo). Al finalizar la unidad, se dedica una semana a intensificar estas actividades (Semana Limpia, Semana de la Alimentación, Semana ecológica)

Para concluir, se realiza un acto en el que participa el ciclo completo y en donde se lleva a cabo una entrega de diplomas de los concursos organizados en el aula y una dramatización alusiva al tema (guiñol o teatro) o un juego colectivo.

\section{Bibliografía}

- Por una Educación Intercultural. Ana Amorós Puente y otros. 1993. MEC

- La escuela multicultural. José Antonio Jordán. 1994. Paidós.

- Educación multicultural. Su teoría y su práctica. Maria Teresa Anguado Odina. 1996. UNED

- Educación Intercultural. Análisis y resolución de conflictos. Colectivo AMANI.1994. Popular. 